\title{
Synthesis of xanthine derivatives by microwave-assisted ring closure reaction
}

\author{
Joachim C. Burbiel,* Jörg Hockemeyer, and Christa E. Müller \\ Pharmazeutisches Institut, Rheinische Friedrich-Wilhelms-Universität Bonn, Kreuzbergweg 26, \\ D-53115 Bonn, Germany \\ E-mail: joachim.burbiel@uni-bonn.de
}

(received 24 Nov 05; accepted 20 Dec 05; published on the web 30 Dec 05)

\begin{abstract}
Ring closure of the imidazole ring is a key step in the synthesis of xanthine and other purine derivatives. A microwave-assisted procedure involving triethyl orthoformate is presented. High yields are achieved even on a gram scale, while reaction times are considerably shortened compared to conventional heating conditions.
\end{abstract}

Keywords: Microwave-assisted synthesis, xanthines, purines, imidazole ring closure

\section{Introduction}

The plant alkaloid caffeine, 1,3,7-trimethylxanthine, is the most frequently used psychostimulant drug world-wide. It exerts its effects by the blockade of adenosine receptors in the brain. ${ }^{1}$ Theophylline, 1,3-dimethylxanthine, which is also naturally occurring, is therapeutically applied as an anti-asthmatic drug. A large number of derivatives and analogs of caffeine and theophylline have been synthesized and pharmacologically investigated. ${ }^{2,3}$ Some xanthine derivatives, including denbufylline and pentoxifylline, are potent phosphodiesterase inhibitors. ${ }^{4-6}$ The latter is clinically used for the treatment of peripheral vascular disease. ${ }^{6}$ Other xanthine derivatives, such as lisofylline, are experimental anti-inflammatory drugs. ${ }^{7}$ Further xanthine derivatives are currently in pre-clinical or clinical development as novel drugs for various indications and many xanthine derivatives have become important pharmacological tools. ${ }^{8}$ It is thus justified to claim that the xanthine scaffold belongs to the so-called "privileged structures" 9 in medicinal chemistry and drug development.

A key step in the classical synthetic route towards xanthines is the ring closure of the imidazole ring, which leads to the purine ring system. The classical Traube synthesis of 8unsubstituted xanthine derivatives comprises the reaction of a 5,6-diaminouracil derivative with formic acid in a two step procedure, involving tedious workup of the intermediate formamide. $^{10,11}$ 
<smiles>CCCCn1c(=O)c2c(ncn2CC(C)=O)n(CCCC)c1=O</smiles><smiles>CC(=O)CCCCn1c(=O)c2c(ncn2C)n(C)c1=O</smiles>

Pentoxifyline<smiles>C[C@H](O)CCCCn1c(=O)c2c(ncn2C)n(C)c1=O</smiles>

Lisofylline

Scheme 1. Examples of xanthine drugs.

As an alternative, a very efficient ring-closure reaction was first described by Grundmann and Kreutzberger. ${ }^{12}$ The reaction of 1,3-dimethyl-4,5-diaminouracil with 1,3,5-triazine led to very pure theophylline in quantitative yield after a reaction time of less than one minute. Although this finding appeared to be very encouraging, its applicability is limited since 1,3,5triazine is not a readily accessible reagent.

Another ring closure procedure is the reaction of 5,6-diaminouracils with triethyl orthoformate, which is easily accessible and requires no special precautions in handling, as it is relatively stable towards hydrolysis. A disadvantage of this method are long reaction times of up to several hours. ${ }^{13,14}$ Poor solubility of the precursors in triethyl orthoformate appears to be one of the problems. The present study was aimed at developing a fast, easy and general method for imidazole ring formation.

\section{Results and Discussion}

We were especially interested in a facile synthesis of 1,3-diethylxanthine 1, which was required as a reaction intermediate in our investigations on the structure-activity relationship of adenosine receptor antagonists, and 1,3-dibutylxanthine $\mathbf{2}$ as a precursor to denbufylline. As starting materials the 1,3-dialkyl-5,6-diaminouracils 3 and $\mathbf{4}$ were prepared as previously described. ${ }^{10}$ In order to obtain highly pure compounds separation methods for these air- and temperature- 
sensitive materials had to be developed (see Experimental), since no detailed procedures are given in the literature. Although the subsequent reactions work well with impure starting materials (containing small quantities of water, inorganic salts or other by-products of the synthesis), highly pure 5,6-diaminouracils were required for the precise determination of the yields.<smiles>[R]n1c(N)c(N)c(=O)n([R])c1=O</smiles>

$$
3 \mathrm{R}^{1}=\mathrm{R}^{3}=\mathrm{Et}
$$$$
4 \mathrm{R}^{1}=\mathrm{R}^{3}=\mathrm{Bu}
$$$$
5 \mathrm{R}^{1}=\mathrm{H}, \mathrm{R}^{3}=\mathrm{Bu}
$$$$
6 \mathrm{R}^{1}=\mathrm{H}, \mathrm{R}^{3}=2
$$

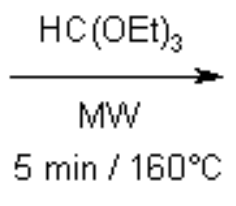

$$
5 \min / 160^{\circ} \mathrm{C}
$$

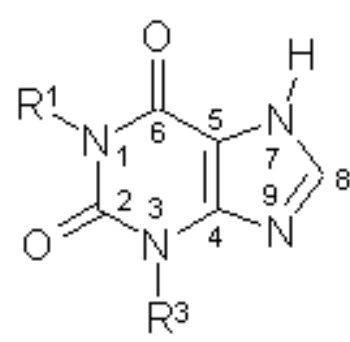

$1 \mathrm{R}^{1}=\mathrm{R}^{3}=\mathrm{Et}$

$2 \mathrm{R}^{1}=\mathrm{R}^{3}=\mathrm{Bu}$

$7 \mathrm{R}^{1}=\mathrm{H}, \mathrm{R}^{3}=\mathrm{Bu}$

$8 R^{1}=H, R^{3}=\longrightarrow$

Scheme 2. Microwave-assisted preparation of xanthine derivatives.

Preliminary experiments had shown that 5,6-diamino-1,3-dibutyluracil 4 was quite reactive towards triethyl orthoformate under reflux conditions, giving an $80 \%$ yield of 1,3dibutylxanthine 2 after one hour. Microwave irradiation sped up the reaction considerably: instead of one hour, the reaction could be completed within 5 minutes with a similar yield. The analogous microwave-assisted reaction with 5,6-diamino-1,3-diethyluracil 3 led to the corresponding 1,3-diethylxanthine 1 in $76 \%$ yield equally fast.

To test the scope of our microwave-assisted method, we also investigated the reaction of the poorly soluble derivatives 1-butyl-5,6-diaminouracil 5 and 5,6-diamino-1-propargyluracil 6 in triethyl orthoformate. Reaction times of $5 \mathrm{~h}$ for the preparation of 7 and $1.5 \mathrm{~h}$ for 8 have been reported for conventional reaction conditions. ${ }^{13}$ After $5 \mathrm{~min}$ of microwave irradiation no residual starting material could be detected. The corresponding 8-unsubstituted xanthine derivatives 7 and 8 were isolated in excellent yields of up to $90 \%$ depending on the purity of the employed 5,6diaminouracils. To test whether this acceleration was only due to the higher temperature applied, a sample of 5,6-diamino-1-propargyluracil 6 was mixed with triethyl orthoformate and conventionally heated at $160^{\circ} \mathrm{C}$ in a glass pressure tube for 10 minutes. After workup, most of the starting material could be recovered, while only traces of xanthine 8 were detected by TLC. 


\section{Table 1}<smiles>[R]n1c(=O)c2[nH]cnc2n([R])c1=O</smiles>

\begin{tabular}{|c|c|c|c|c|c|}
\hline \multirow[t]{2}{*}{ Xanthine } & \multirow[t]{2}{*}{$\mathrm{R}^{1}$} & \multirow[t]{2}{*}{$\mathrm{R}^{3}$} & \multicolumn{2}{|c|}{ Reaction time (min) } & \multirow{2}{*}{$\begin{array}{l}\text { Yield (\%) } \\
\text { Method B }\end{array}$} \\
\hline & & & Method $\mathrm{A}^{\mathrm{a}}$ & Method B ${ }^{b}$ & \\
\hline 1 & Et & Et & n.d. & 5 & 76 \\
\hline 2 & $\mathrm{Bu}$ & $\mathrm{Bu}$ & 60 & 5 & 80 \\
\hline 7 & $\mathrm{Bu}$ & $\mathrm{H}$ & $300^{\mathrm{c}}$ & 5 & 85 \\
\hline 8 & propargyl & $\mathrm{H}$ & $90^{\mathrm{c}}$ & 5 & 90 \\
\hline
\end{tabular}

${ }^{a}$ Heating with triethyl orthoformate under reflux.

${ }^{\mathrm{b}}$ Heating with triethyl orthoformate at $160^{\circ} \mathrm{C}$ with microwave irradiation.

${ }^{\mathrm{c}}$ Taken from the literature ${ }^{13}$.

Notably, the reactions proceeded smoothly, although the employed quantity of triethyl orthoformate was far too little to dissolve either the starting materials or the products. This greatly facilitated purification and workup, especially when working on a gram scale. We were thus able to drastically shorten the reaction times in the synthesis of xanthines using triethyl orthoformate by applying microwave energy. This reaction seems to have a broad scope concerning substitution patterns at the 1- and 3-position and is thus an easy and convenient procedure to synthesize 8 -unsubstituted xanthine derivatives.

\section{Experimental Section}

General Procedures. All microwave reactions were carried out in $10 \mathrm{ml}$ sealed glass tubes in a focused mono-mode microwave oven ("Discover" by CEM Corporation, Matthews, NC). Maximum power levels, target temperatures and reaction times are given. All commercially available reagents and solvents were used without further purification. Petrol ether with a boiling point of $40-60^{\circ} \mathrm{C}$ was used, if not otherwise noted. The melting points were measured by a Wepa "apotec" melting point apparatus and are uncorrected. ${ }^{1} \mathrm{H}$ and ${ }^{13} \mathrm{C}$ NMR spectra were performed on a Bruker Avance $500 \mathrm{MHz}$ spectrometer. The chemical shifts of the remaining protons of the deuterated solvent served as internal standard. Mass spectra were recorded on a MS-50 spectrometer at the Chemical Institute, University of Bonn.

5,6-Diamino-1,3-dialkyluracils ${ }^{10}$. In $60 \mathrm{~mL}$ of $12.5 \%$ aq. ammonia solution, $20 \mathrm{mmol}$ of the corresponding 1,3-dialkyl-6-amino-5-nitrosouracil ${ }^{10}$ was dissolved. The solution was kept at 50- 
$60{ }^{\circ} \mathrm{C}$, and $\mathrm{Na}_{2} \mathrm{~S}_{2} \mathrm{O}_{4}$ (ca. 9 g) was added upon stirring until the originally deeply colored solution had almost decolorized. After cooling to RT, ca. $100 \mathrm{ml}$ of brine was added and the solution was extracted four times, each time with $100 \mathrm{~mL}$ of $\mathrm{CH}_{2} \mathrm{Cl}_{2}$. The organic extracts were dried over $\mathrm{MgSO}_{4}$, filtered and concentrated. Compound 3 was dissolved in $\mathrm{CH}_{2} \mathrm{Cl}_{2}$, precipitated by adding petrol ether, filtered under reduced pressure and washed with petrol ether. Compound 4 was purified by column chromatography on silica gel (eluent: $\mathrm{CH}_{2} \mathrm{Cl}_{2}$ : ethanol = 9:1). The productcontaining fractions were concentrated and treated as described for compound 3. The isolated diaminouracils were dried and stored in a vacuum desiccator at RT.

5,6-Diamino-1,3-diethyluracil (3). Yield: 3.2 g (80\%); mp 103-104 ${ }^{\circ} \mathrm{C} .{ }^{1} \mathrm{H}$ NMR (500 MHz, $\left.\mathrm{CDCl}_{3}\right) \delta 1.18\left(\mathrm{t}, 3 \mathrm{H}, J=7.25 \mathrm{~Hz}, \mathrm{CH}_{3}\right), 1.29\left(\mathrm{t}, J=7.25 \mathrm{~Hz}, 3 \mathrm{H}, \mathrm{CH}_{3}\right), 2.26$ (br. s, 2H, $\mathrm{NH}_{2}$ ), 3.92 (q, $\left.J=7.25 \mathrm{~Hz}, 2 \mathrm{H}, \mathrm{CH}_{2}\right), 3.96$ (q, $\left.J=7.25 \mathrm{~Hz}, 2 \mathrm{H}, \mathrm{CH}_{2}\right), 4.92\left(\mathrm{~s}, 2 \mathrm{H}, \mathrm{NH}_{2}\right) ;{ }^{13} \mathrm{C} \mathrm{NMR}$ $\left(125 \mathrm{MHz}, \mathrm{CDCl}_{3}\right) \delta 13.2\left(\mathrm{CH}_{3}\right), 13.5\left(\mathrm{CH}_{3}\right), 36.6\left(\mathrm{CH}_{2}\right), 38.2\left(\mathrm{CH}_{2}\right), 95.7(\mathrm{C} 5), 148.5(\mathrm{C} 6)$, 149.9 (C2), 161.4 (C4); EIMS (m/z, \%) $198\left(\mathrm{M}^{+}, 100\right)$.

5,6-Diamino-1,3-dibutyluracil (4). Yield: $2.6 \mathrm{~g}(51 \%)$; mp $93{ }^{\circ} \mathrm{C} .{ }^{1} \mathrm{H} \mathrm{NMR}\left(500 \mathrm{MHz}, \mathrm{CDCl}_{3}\right)$ $\delta 0.90\left(\mathrm{t}, 3 \mathrm{H}, J=7.6 \mathrm{~Hz}, \mathrm{CH}_{3}\right), 0.95\left(\mathrm{t}, J=7.25 \mathrm{~Hz}, 3 \mathrm{H}, \mathrm{CH}_{3}\right), 1.33\left(\mathrm{~m}, 2 \mathrm{H}, \mathrm{CH}_{2}\right), 1.38(\mathrm{~m}, 2 \mathrm{H}$, $\mathrm{CH}_{2}$ ), $1.56\left(\mathrm{~m}, 2 \mathrm{H}, \mathrm{CH}_{2}\right), 1.64\left(\mathrm{~m}, 2 \mathrm{H}, \mathrm{CH}_{2}\right), 2.28$ (br. s, 2H, $\left.\mathrm{NH}_{2}\right), 3.83$ (t, J = $7.9 \mathrm{~Hz}, 2 \mathrm{H}$, $\left.\mathrm{NCH}_{2}\right), 3.89\left(\mathrm{t}, J=7.6 \mathrm{~Hz}, 2 \mathrm{H}, \mathrm{NCH}_{2}\right), 4.87\left(\mathrm{~s}, 2 \mathrm{H}, \mathrm{NH}_{2}\right) ;{ }^{13} \mathrm{C} \mathrm{NMR}\left(125 \mathrm{MHz}, \mathrm{CDCl}_{3}\right) \delta 13.7$ $\left(\mathrm{CH}_{3}\right), 13.8\left(\mathrm{CH}_{3}\right), 20.1\left(\mathrm{CH}_{2}\right), 20.2\left(\mathrm{CH}_{2}\right), 30.1\left(\mathrm{CH}_{2}\right), 30.4\left(\mathrm{CH}_{2}\right), 41.4\left(\mathrm{CH}_{2}\right), 43.1\left(\mathrm{CH}_{2}\right), 95.7$ (C5), 148.5 (C6), 150.3 (C6), 161.6 (C4); EIMS (m/z, \%) $254\left(\mathrm{M}^{+}, 100\right)$.

\section{1,3-Dialkylxanthines 1 and 2}

A mixture of $1 \mathrm{~g}$ of 5,6-diamino-1,3-dialkyluracil (5 mmol of 3 or $4 \mathrm{mmol}$ of 4, respectively) and triethyl orthoformate $(6 \mathrm{ml})$ was subjected to microwave irradiation for $5 \mathrm{~min}\left(120 \mathrm{~W}, 160^{\circ} \mathrm{C}\right)$ in a $10 \mathrm{ml}$ pressure tube, with stirring. The resulting yellow suspension was diluted with $50 \mathrm{ml}$ of 2 $\mathrm{N}$ aq. $\mathrm{HCl}$ solution. After the addition of brine, the aqueous suspension was extracted three times with dichloromethane $\left(100 \mathrm{ml}\right.$ each). The united organic phases were dried over $\mathrm{MgSO}_{4}$ and concentrated under reduced pressure. The products were dissolved in $\mathrm{CH}_{2} \mathrm{Cl}_{2}$, precipitated by adding petrol ether, filtered off, washed with petrol ether $(10 \mathrm{ml})$ and dried at $70{ }^{\circ} \mathrm{C}$.

1,3-Diethylxanthine (1). Yield: $0.79 \mathrm{~g}$ colorless crystals $(76 \%)$; mp $221{ }^{\circ} \mathrm{C}\left(\mathrm{Lit}^{15} 212{ }^{\circ} \mathrm{C}\right) .{ }^{1} \mathrm{H}-$ $\operatorname{NMR}\left(500 \mathrm{MHz}, \mathrm{CDCl}_{3}\right) \delta=1.28\left(\mathrm{t}, 3 \mathrm{H}, J=7.1 \mathrm{~Hz}, \mathrm{CH}_{3}\right), 1.36\left(\mathrm{t}, J=7.35 \mathrm{~Hz}, 3 \mathrm{H}, \mathrm{CH}_{3}\right), 4.15$ (q, 2H, J=7.1 Hz, $\mathrm{CH}_{2}$ ), 4.21 (q, 2H, J = 7.1 Hz, $\left.\mathrm{CH}_{2}\right), 7.81\left(\mathrm{~d}, 1 \mathrm{H}, J=1.6 \mathrm{~Hz}, \mathrm{C}_{8} \mathrm{H}\right), 12.77$ (s, $1 \mathrm{H}, \mathrm{NH}) ;{ }^{13} \mathrm{C} \mathrm{NMR}\left(125 \mathrm{MHz}, \mathrm{CDCl}_{3}\right) \delta 13.3\left(\mathrm{CH}_{3}\right), 13.4\left(\mathrm{CH}_{3}\right), 37.0\left(\mathrm{CH}_{2}\right), 39.0\left(\mathrm{CH}_{2}\right), 107.1$ (C5), 140.3, 148.5, 150.5, 156.1; EIMS (m/z, \%) $208\left(\mathrm{M}^{+}, 100\right)$.

1,3-Dibutylxanthine (2). Yield: 0.85 g colorless crystals $(80 \%)$; mp 186-187 ${ }^{\circ} \mathrm{C}$ (Lit. $\left.{ }^{10} 189{ }^{\circ} \mathrm{C}\right)$. ${ }^{1} \mathrm{H}-\mathrm{NMR}\left(500 \mathrm{MHz}, \mathrm{CDCl}_{3}\right) \delta=0.94\left(\mathrm{t}, 3 \mathrm{H}, J=7.55 \mathrm{~Hz}, \mathrm{CH}_{3}\right), 0.95\left(\mathrm{t}, J=7.25 \mathrm{~Hz}, 3 \mathrm{H}, \mathrm{CH}_{3}\right)$, $1.38\left(\mathrm{~m}, 2 \mathrm{H}, \mathrm{CH}_{2}\right), 1.40\left(\mathrm{~m}, 2 \mathrm{H}, \mathrm{CH}_{2}\right), 1.65\left(\mathrm{~m}, 2 \mathrm{H}, \mathrm{CH}_{2}\right), 1.75\left(\mathrm{~m}, 2 \mathrm{H}, \mathrm{CH}_{2}\right), 4.08(\mathrm{~m}, 2 \mathrm{H}$, $\left.\mathrm{NCH}_{2}\right), 4.13\left(\mathrm{~m}, 2 \mathrm{H}, \mathrm{NCH}_{2}\right), 7.78\left(\mathrm{~d}, 1 \mathrm{H}, J=1.3 \mathrm{~Hz}, \mathrm{C}_{8} \mathrm{H}\right), 13.00(\mathrm{~s}, 1 \mathrm{H}, \mathrm{NH}) ;{ }^{13} \mathrm{C}$ NMR $(125$ $\left.\mathrm{MHz}, \mathrm{CDCl}_{3}\right) \delta 13.7\left(\mathrm{CH}_{3}\right), 13.8\left(\mathrm{CH}_{3}\right), 20.0\left(\mathrm{CH}_{2}\right), 20.2\left(\mathrm{CH}_{2}\right), 30.1\left(\mathrm{CH}_{2}\right), 30.1\left(\mathrm{CH}_{2}\right), 41.6$ $\left(\mathrm{CH}_{2}\right), 43.8\left(\mathrm{CH}_{2}\right), 107.0(\mathrm{C} 5), 140.3,148.8,150.3,156.3$; EIMS (m/z, \%) $264\left(\mathrm{M}^{+}, 100\right)$. 
1-Alkylxanthines 7 and $8^{13}$. A mixture of $1 \mathrm{~g}$ of 5,6-diamino-3-alkyluracil (5 mmol of $5^{13}$ or 5.5 mmol of $\mathbf{6}^{13}$, respectively) and triethyl orthoformate $(6 \mathrm{ml})$ was subjected to microwave irradiation for $5 \mathrm{~min}\left(120 \mathrm{~W}, 160^{\circ} \mathrm{C}\right)$ in a $10 \mathrm{ml}$ pressure tube upon stirring. The products were filtered off, washed with diethyl ether $(10 \mathrm{ml})$ and recrystallized from water.

1-Butylxanthine (7). Yield: $0.88 \mathrm{~g}$ colorless crystals $(85 \%)$; mp $261{ }^{\circ} \mathrm{C}$ (Lit. $\left.{ }^{13} 262{ }^{\circ} \mathrm{C}\right)$. TLC control: comparison with a conventionally prepared sample.

1-Propargylxanthine (8). Yield: 0.94 g colorless crystals $(90 \%)$; mp $278{ }^{\circ} \mathrm{C}$ (Lit. $\left.{ }^{13} 279{ }^{\circ} \mathrm{C}\right)$. TLC control: comparison with a conventionally prepared sample.

\section{References}

1. Daly, J. W.; Fredholm, B. B. Drug Alcohol Depend. 1998, 51, 199.

2. Daly, J. W. Isn Role of adenosine and adenine nucleotides in the biological system, Imai, S.; Nakazawa, M., Eds., Elsevier Science Publishers: Amsterdam, 1991; pp 119-129.

3. Stefanovich, V. Drug News Perspect. 1989, 2, 82.

4. Nicholson, C. D.; Jackman, S. A.; Wilke, R. Br. J. Pharmacol. 1989, 97, 889.

5. Thiel, M.; Bardenheuer, H.; Poech, G.; Madel, C.; Peter, K. Biochem. Res. Commun. 1991, 180, 53.

6. Palacios, J. M.; Beleta, J.; Segarra, V. Farmaco 1995, 50, 819.

7. Bright, J. J.; Du, C.; Coon, M.; Sriram, S.; Klaus, S. J. J. Immunol. 1998, 161, 7051.

8. Daly, J. W. J. Auton. Nerv. Syst. 2000, 81, 44.

9. Evans, B. E.; Rittle, K. E.; Bock, M. G.; DiPardo, R. M.; Freidinger, R. M.; Whitter, W. L.; Lundell, G. F.; Verber, D. F.; Anderson, P. S. J. Med. Chem. 1988, 31, 2235.

10. Speer, J.; Raymond, A. J. Am. Chem. Soc. 1953, 75, 114.

11. Kim, H.; Ji, X.; Melman, N.; Olah, M.; Stiles, G.; Jacobson, K. J. Med. Chem. 1994, 37, 3373.

12. Grundmann, C.; Kreutzberger, A. J. Am. Chem. Soc. 1955, 77, 6559.

13. Müller, C. E. Synthesis 1993, 125.

14. Kim, S.-A.; Marshall, M. A.; Melman, N.; Kim, H. S.; Müller, C. E.; Linden, J.; Jacobson, K. A. J. Med. Chem. 2002, 45, 2131.

15. Ruttink, J. Recl. Trav. Chim. Pays-Bas 1946, 65, 751. 